INTER NATIONAL MONETARY FUND

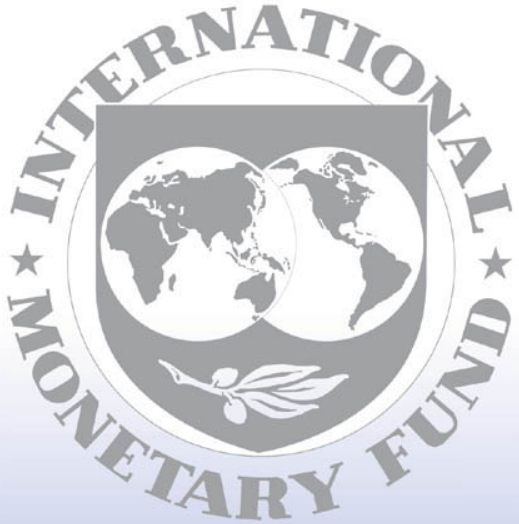

Staff

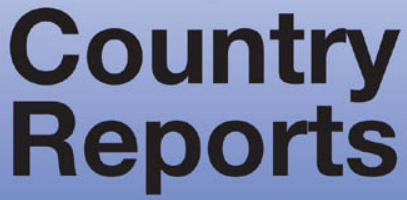




\section{Paraguay: Statistical Appendix}

This Statistical Appendix paper for Paraguay was prepared by a staff team of the International Monetary Fund as background documentation for the periodic consultation with the member country. It is based on the information available at the time it was completed on February 26, 2003. The views expressed in this document are those of the staff team and do not necessarily reflect the views of the government of Paraguay or the Executive Board of the IMF

The policy of publication of staff reports and other documents by the IMF allows for the deletion of market-sensitive information.

To assist the IMF in evaluating the publication policy, reader comments are invited and may be sent by e-mail to publicationpolicy@imf.org.

Copies of this report are available to the public from

International Monetary Fund $\bullet$ Publication Services $70019^{\text {th }}$ Street, N.W. • Washington, D.C. 20431

Telephone: (202) 623-7430 • Telefax: (202) 623-7201

E-mail: publications@imf.org • Internet: http://www.imf.org

Price: $\$ 15.00$ a copy

International Monetary Fund

Washington, D.C. 


\section{INTERNATIONAL MONETARY FUND}

\section{PARAGUAY}

\section{Statistical Appendix}

Prepared by Jeffrey Franks, Valerie Mercer-Blackman, Randa Sab, and Chad Steinberg (all WHD), and Ward Brown (PDR)

Approved by Western Hernisphere Department

February 26, 2003

Contents

I. Real Sector

1. National Accounts in Current Prices .3

2. National Accounts in Constant Prices .............................................................

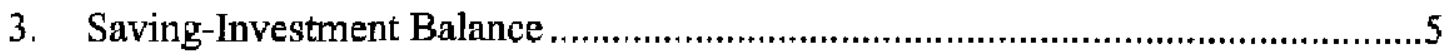

4. GDP by Sector at Current Prices .................................................................

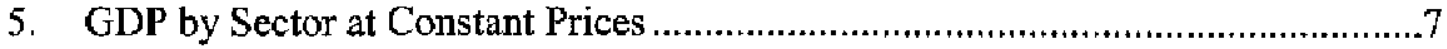

6. Value of Agricultural Production ................................................................

7. Production, Area Cultivated, and Yields of Selected Crops ............................... 9

8. Value-Added in Manufacturing...................................................................10

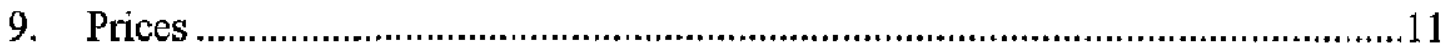

10. Labor Market Indicators …….....................................................................12

II. Public Sector

11. Operations of the Consolidated Public Sector ..................................................13

12. Operations of the Central Government ...........................................................14

13. Operations of the Social Security Institute (IPS) ...............................................15

14. Rest of the General Government .................................................................16

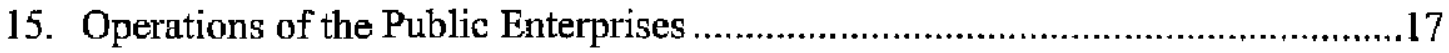

16. Quasi-Fiscal Operations ...............................................................................18

III. Monetary Sector

17. Central Bank Balance Sheet .......................................................................19

18. Commercial Banks Balance Sheet..................................................................20

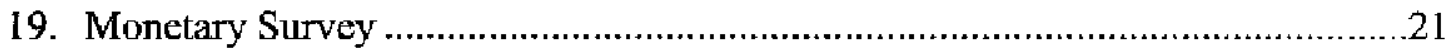

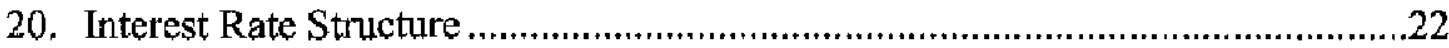

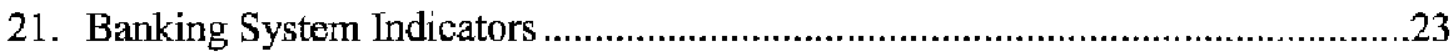


IV. External Sector

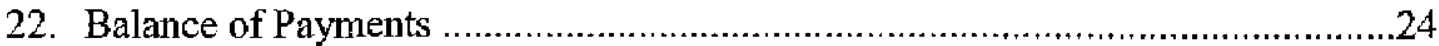

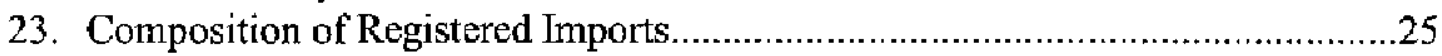

24. Composition of Registered Exports .........................................................26

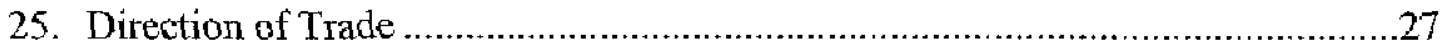


Table 1. Paraguay: National Accounts in Current Prices

\begin{tabular}{|c|c|c|c|c|}
\hline & 1998 & 1999 & 2000 & $\begin{array}{l}\text { Prel. } \\
2001\end{array}$ \\
\hline \multicolumn{5}{|c|}{ (In billions of guaranies) } \\
\hline GDP at market prices & $23,437.0$ & $24,144.3$ & $26,921.0$ & $28,118.8$ \\
\hline Growth & 12.0 & 3.0 & 11.5 & 4.4 \\
\hline Consumption expenditure & $19,009.2$ & $19,695.2$ & $22,275.2$ & $23,893.6$ \\
\hline Private sector & $16,163.8$ & $16,772.7$ & $18,688.8$ & $20,370.8$ \\
\hline Public sector & $2,845.4$ & $2,922.6$ & $3,586.5$ & $3,522.8$ \\
\hline Gross domestic investment & $5,373.8$ & $5,563.7$ & $5,881.1$ & $5,560.6$ \\
\hline Private sector & $3,658.3$ & $2,857.1$ & $4,000.1$ & $3,869.4$ \\
\hline Public sector & $1,715.5$ & $2,706.6$ & $1,881.0$ & $1,691.2$ \\
\hline Fixed capital formation & $5,168.2$ & $5,342.1$ & $5,638.6$ & $5,295.0$ \\
\hline Changes in inwentories & 205.6 & 221.7 & 242.5 & 265.5 \\
\hline Gross domestic expenditure & $24,383.0$ & $25,259.0$ & $28,156.3$ & $29,454.1$ \\
\hline Net exports & -946.0 & $-1,114.7$ & $-1,235.3$ & $-1,335.3$ \\
\hline Exports of goods and nonfactor services & $11,502.7$ & $8,980.4$ & $10,248.1$ & $12,471.1$ \\
\hline Imports of goods and nonfactor services & $-12,448.8$ & $-10,095.1$ & $-11,483.4$ & $-13,806,4$ \\
\hline \multicolumn{5}{|c|}{ (In percent of GDP) } \\
\hline GDP at market prices & 100.0 & 100.0 & 100.0 & 100.0 \\
\hline Consumption expenditure & 81.1 & 81.6 & 82.7 & 85.0 \\
\hline Private sector & 69.0 & 69.5 & 69.4 & 72.4 \\
\hline Public sector & 12.1 & 12.1 & 13.3 & 12.5 \\
\hline Gross domestic investment & 22.9 & 23.0 & 21.8 & 19.8 \\
\hline Private sector & 15.6 & 11.8 & 14.9 & 13.8 \\
\hline Public sector & 7.3 & 11.2 & 7.0 & 6.0 \\
\hline Fixed capital formation & 22.1 & 22.1 & 20.9 & 18.8 \\
\hline Changes in inventories & 0.9 & 0.9 & 0.9 & 0.9 \\
\hline Gross domestic expenditure & 104.0 & 104.6 & 104.6 & 104.7 \\
\hline Net exports & -4.0 & -4.6 & -4.6 & -4.7 \\
\hline Exports of goods and nonfactor services & 49.1 & 37.2 & 38.1 & 44.4 \\
\hline Imports of goods and nonfactor services & -53.1 & -41.8 & -42.7 & -49.1 \\
\hline
\end{tabular}

Sources: Central Bank of Paraguay; and Fund staff estimates. 
Table 2. Paraguay: National Accounts in Constant Prices

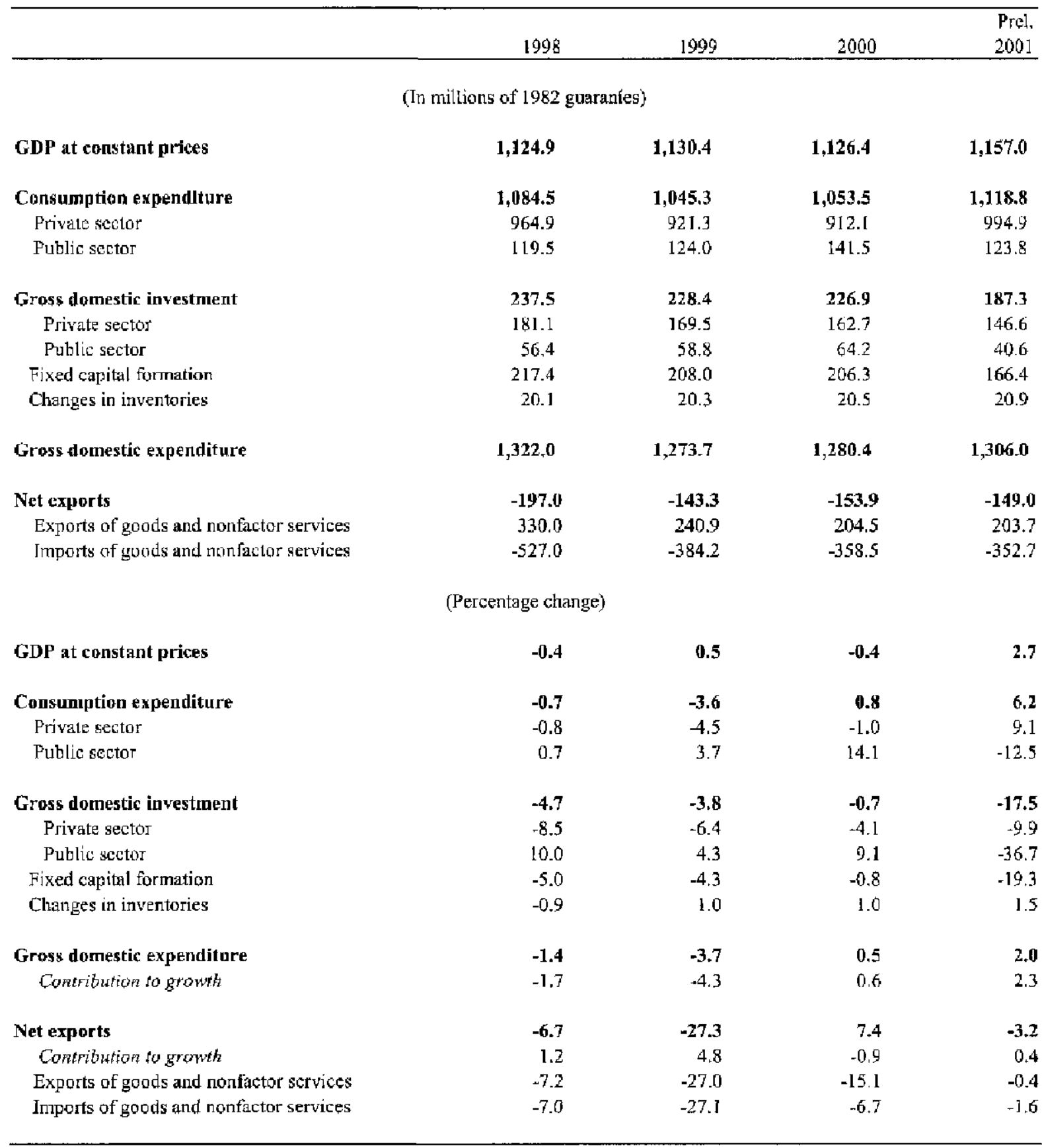

Sources: Central Bank of Paraguay; and Fund staff estimates. 
Table 3. Paraguay: Savings-Investment Balance

(In percent of GDP)

\begin{tabular}{|c|c|c|c|c|}
\hline & 1998 & 1999 & 2000 & $\begin{array}{l}\text { Prel, } \\
2001\end{array}$ \\
\hline GDP at market prices & 100.0 & 100.0 & 100.0 & 100.0 \\
\hline Domestic expenditure & 104.0 & 104.6 & 104.6 & 104.7 \\
\hline Consumption & 81.1 & 81.6 & 82.7 & 85.0 \\
\hline Private sector & 69.0 & 69.5 & 69.4 & 72.4 \\
\hline Public sector & 12.1 & 12.1 & 13.3 & 12.5 \\
\hline Gross domestic investment & 22.9 & 23.0 & 21.8 & 19.8 \\
\hline Private sector & 15.6 & 11.8 & 14.9 & 13.8 \\
\hline Public sector & 7.3 & 11.2 & 7.0 & 6.0 \\
\hline Gross national savings & 21.1 & 20.9 & 19.9 & 17.5 \\
\hline Private sector & 13.5 & 12.9 & 17.0 & 12.4 \\
\hline Public sector & 7.5 & 8.0 & 2.9 & 5.1 \\
\hline Current account balance & -1.8 & -2.1 & -1.9 & -2.3 \\
\hline Private savings-investment balance & -2.1 & 1.0 & 2.1 & -1.3 \\
\hline Public sector overall balance & 0.2 & -3.2 & -4.1 & -0.9 \\
\hline
\end{tabular}

Sources: Central Bank of Paraguay; and Fund staff estimates. 
Table 4. Paraguay: GDP by Sector at Current Prices

\begin{tabular}{|c|c|c|c|c|}
\hline & 1998 & 1999 & 2000 & $\begin{array}{l}\text { Prel. } \\
2001 \\
\end{array}$ \\
\hline \multicolumn{5}{|c|}{ (In billions of guaranies) } \\
\hline GDP at market prices & $23,437.0$ & $24,144.3$ & $26,921.0$ & $28,118.8$ \\
\hline Primary sector & $5,795.9$ & $6,156.7$ & $6,861.3$ & $6,748.9$ \\
\hline Agriculture & $3,492.4$ & $3,714.6$ & $4,180.9$ & $4,000.6$ \\
\hline Livestock & $1,470.1$ & {$[, 563.6$} & $1,747.1$ & 1.813 .2 \\
\hline Forestry & 725.0 & 767.1 & 812.2 & 818.0 \\
\hline Hunting and fishing & 28.8 & 22.3 & 23.8 & 31.2 \\
\hline Mining & 79.5 & 89.0 & 97.2 & 85.8 \\
\hline Secondary sector & $6,213.4$ & $6,169.9$ & $6,940.0$ & $7,105.7$ \\
\hline Manufacturing & $3,631.5$ & $3,272.1$ & $3,689.2$ & $4,011.6$ \\
\hline Construction & $1,433.1$ & $1,502.9$ & $1,615.1$ & $1,310.9$ \\
\hline Utilities & $1,148.9$ & $1,394.9$ & $1,635.7$ & $1,783.2$ \\
\hline Services & $11,427.6$ & $11,817.7$ & $13,119.7$ & $14,264.3$ \\
\hline Transport and communications & 913.9 & $1,063.4$ & $1,303.2$ & $1,486.1$ \\
\hline Commerce and finance & $6,309.9$ & $5,887.1$ & $6,250.4$ & $6,772.5$ \\
\hline General government & $1,181.8$ & $1,360.3$ & $1,537.8$ & $1,588.5$ \\
\hline Real estate and housing & 515.8 & 561.5 & 610.1 & 687.6 \\
\hline Other services & $2,506.2$ & $2,945.3$ & $3,418.3$ & $3,729.5$ \\
\hline \multicolumn{5}{|c|}{ (In percent of GDP) } \\
\hline GDP at market prices & 100.0 & 100.0 & 100.0 & 100.0 \\
\hline Primary sector & 24.7 & 25.5 & 25.5 & 24.0 \\
\hline Agriculture & 14.9 & 15.4 & 15.5 & 14.2 \\
\hline Livestock & 6.3 & 6.5 & 6.5 & 6.4 \\
\hline Forestry & 3.1 & 3.2 & 3.0 & 2.9 \\
\hline Hutrting and fishing & 0.1 & 0.1 & 0.1 & 0.1 \\
\hline Mining & 0.3 & 0.4 & 0.4 & 0.3 \\
\hline Secondiry sector & 26.5 & 25.6 & 25.8 & 25.3 \\
\hline Manufacturing & 15.5 & 13.6 & 13.7 & 14.3 \\
\hline Construction & 6.1 & 6.2 & 6.0 & 4.7 \\
\hline Utilities & 4.9 & 5.8 & 6.1 & 6.3 \\
\hline Services & 48.8 & 48.9 & 48.7 & 50.7 \\
\hline Transport ard communications & 3.9 & 4.4 & 4.8 & 5.3 \\
\hline Commerce and finance & 26.9 & 24.4 & 23.2 & 24.1 \\
\hline General government & 5.0 & 5.6 & 5.7 & 5.6 \\
\hline Real estate and housing & 2.2 & 2.3 & 2.3 & 2.4 \\
\hline Other services & 10.7 & 12.2 & 12.7 & 13.3 \\
\hline
\end{tabular}

Sources: Central Bank of Paraguay; and Fund staff estimates. 
Table 5. Paraguay: GDP by Sector at Constant Prices

\begin{tabular}{|c|c|c|c|c|}
\hline & 1998 & 1999 & 2000 & $\begin{array}{l}\text { Prel. } \\
2001\end{array}$ \\
\hline \multicolumn{5}{|c|}{ (In millions of 1982 guaranies) } \\
\hline GDP at constant prices & $1,124.9$ & $1,130.4$ & $1,126.4$ & $1,157.0$ \\
\hline Primary sector & 313.2 & 323.1 & 309.3 & $\mathbf{3 4 0 . 4}$ \\
\hline Agriculture & 188.1 & 195.6 & 179.0 & 212.4 \\
\hline Livestock & 87.2 & 89.1 & 90.9 & 90.3 \\
\hline Forestry & 31.1 & 31.3 & 32.2 & 31.3 \\
\hline Hunting and fishing & 1,6 & 1.6 & 1.6 & 1.6 \\
\hline Mining & 5.4 & 5.5 & 5.6 & 4.8 \\
\hline Secondary sector & 283.9 & 294.9 & 303.5 & 291.7 \\
\hline Manufacturing & 159.0 & 159.1 & 160.7 & 162.8 \\
\hline Construction & 61.0 & 62.5 & 63.8 & 49.3 \\
\hline Utilities & 63.9 & 73.3 & 79.0 & 79.5 \\
\hline Services & 527.8 & 512.5 & 513.5 & 525.0 \\
\hline Transport and communications & 55.8 & 57.2 & 63.5 & 68.9 \\
\hline Commerce and finance & 268.5 & 243.9 & 231.9 & 237,6 \\
\hline General government & 63.6 & 67.7 & 70.6 & 68.3 \\
\hline Real estate and housing & 31.3 & 31.9 & 32.5 & 32.8 \\
\hline Other services & 108.6 & 111.7 & 115.0 & 117.3 \\
\hline \multicolumn{5}{|c|}{ (Percentage change) } \\
\hline GDP at constant prices & -0.4 & 0.5 & -0.4 & 2.7 \\
\hline Primary sector & 0.3 & 3.1 & -4.3 & 10.0 \\
\hline Agriculture & 0.7 & 4.0 & -8.5 & 18.7 \\
\hline Livestock & -0.8 & 2.2 & 2.0 & -0.7 \\
\hline Forestry & 0.2 & 0.7 & 3.0 & -3.0 \\
\hline Hunting and fishing & 2.0 & 2.0 & 2.0 & 1.5 \\
\hline Mining & 2.5 & 2.2 & 2.5 & -15.2 \\
\hline Secondary sector & 1.1 & 3.9 & 2.9 & -3.9 \\
\hline Manufacturing & 1.0 & 0.0 & 1,0 & 1.3 \\
\hline Construction & 1.0 & 2.5 & 2,0 & -22.7 \\
\hline Utilities & 1.6 & 14.7 & 7.8 & 0.7 \\
\hline Services & -1.6 & $-2,9$ & 0.2 & 2.2 \\
\hline Transport and communications & 3.0 & 2.6 & 10.9 & 8.6 \\
\hline Commerce and finance & -4.2 & -9.2 & -4.9 & 2.4 \\
\hline General government & -2.0 & 6.5 & 4.2 & -3.3 \\
\hline Real estate and housing & 2.0 & 2.0 & 2.0 & 1.0 \\
\hline Other services & 2.0 & 2.8 & 3.0 & 2.0 \\
\hline
\end{tabular}

Sources: Central Bank of Paraguay; and Fund staff estimates. 
Table 6. Paraguay: Value of Agricultural Production 1/

\begin{tabular}{|c|c|c|c|c|}
\hline & 1998 & 1999 & 2000 & $\begin{array}{l}\text { Prel, } \\
2001 \\
\end{array}$ \\
\hline \multicolumn{5}{|c|}{ (In millions of guaranies) } \\
\hline Total & $3,880,497$ & $4,127,348$ & $4,645,445$ & $4,445,080$ \\
\hline Cotton & 201,354 & 175,723 & 235,992 & 248,511 \\
\hline Rice & 41,090 & 67,626 & 42,982 & 49,016 \\
\hline Sugarcane & 103,250 & 109,617 & 93,038 & 107,109 \\
\hline Sunflowers & 42,364 & 41,962 & 59,129 & 20,848 \\
\hline Corn & 315,193 & 528,732 & 434,655 & 351,095 \\
\hline Manioc & 640,200 & 879,220 & $1,125,840$ & 846,411 \\
\hline Peanuts & 30,001 & 34,291 & 37,495 & 22,550 \\
\hline Beans & 32,474 & 55,692 & 50,008 & 71,322 \\
\hline Soybeans & $1,416,448$ & $1,045,661$ & $1,299,316$ & $1,499,218$ \\
\hline Tobacco & 33,934 & 16,307 & 7,775 & 17,924 \\
\hline Wheat & 47,769 & 82,121 & 98,228 & 95,724 \\
\hline Other & 976,420 & $1,090,396$ & $1,160,987$ & $1,115,352$ \\
\hline \multicolumn{5}{|c|}{ (In percent of total) } \\
\hline Cotton & 5.2 & 4.3 & 5.1 & 5.6 \\
\hline Rice & 1.1 & 1.6 & 0.9 & 1.1 \\
\hline Sugarcane & 2.7 & 2.7 & 2.0 & 2.4 \\
\hline Sunflowers & 1.1 & 1.0 & 1.3 & 0.5 \\
\hline Corn & 8.1 & 12.8 & 9.4 & 7.9 \\
\hline Manioc & 16.5 & 21.3 & 24.2 & 19.0 \\
\hline Peanuts & 0.8 & 0.8 & 0.8 & 0.5 \\
\hline Beans & 0.8 & 1.3 & 1.1 & 1.6 \\
\hline Soybeans & 36.5 & 25.3 & 28.0 & 33.7 \\
\hline Tobacco & 0.9 & 0.4 & 0.2 & 0.4 \\
\hline Wheat & 1.2 & 2.0 & 2.1 & 2.2 \\
\hline Other & 25.2 & 26.4 & 25.0 & 25.1 \\
\hline
\end{tabular}

Sounce: Central Bank of Paraguay.

1/ Agricultural years; for example, 1991 refers to the 1990/91 agricultural year. 
Table 7. Paraguay: Production, Area Cultivated, and Yields of Selected Crops 1/

(Production in metric tons; area in hectares; yiclds in $\mathrm{kg} / \mathrm{hcetarc)}$

\begin{tabular}{|c|c|c|c|c|c|}
\hline & 1998 & 1999 & 2000 & 2001 & 2002 \\
\hline \multicolumn{6}{|l|}{ Catton } \\
\hline Area & 202,000 & 166,204 & 194,760 & 297,865 & 169,671 \\
\hline Froduction & 222,000 & 202,283 & 246,583 & 294,444 & 123,667 \\
\hline Yield & 1,099 & 1,217 & 1,266 & 989 & 729 \\
\hline \multicolumn{6}{|l|}{ Rice } \\
\hline Arca & 20,860 & 27,770 & 26,252 & 26,680 & 27,026 \\
\hline Production & 80,921 & 128,093 & 101,049 & 106,179 & 104,973 \\
\hline Yisld & 3,879 & 4,613 & 3,849 & 3,980 & 3,884 \\
\hline \multicolumn{6}{|l|}{ Sugarcane } \\
\hline Area & 58,000 & 61,173 & 59,450 & 59,580 & 60,244 \\
\hline Production & $2,800,000$ & $2,872,271$ & $2,244,900$ & $2,396,180$ & $3,007,212$ \\
\hline Yield & 48,276 & 46,953 & 37,761 & 40,218 & 49,917 \\
\hline \multicolumn{6}{|l|}{ Sunflowers } \\
\hline Area & 62,003 & 52,154 & 70,800 & 30,372 & 25,768 \\
\hline Production & 81,372 & 72,536 & 81,950 & 39,862 & 36,013 \\
\hline Yield & 1,312 & 1,391 & 1,157 & 1,312 & 1,398 \\
\hline \multicolumn{6}{|l|}{ Corn } \\
\hline Arca & 355,600 & 356,602 & 331,725 & 406,365 & 367,960 \\
\hline Production & 873,900 & 817,233 & 647,270 & 947,167 & 867,270 \\
\hline Yield & 2,458 & 2,292 & 1,951 & 2,331 & 2,357 \\
\hline \multicolumn{6}{|l|}{ Manioc } \\
\hline Area & 236,696 & 247,946 & 201,792 & 243,075 & 258,164 \\
\hline Production & $3,300,000$ & $3,694,395$ & $2,719,410$ & $3,368,006$ & $4,430,331$ \\
\hline Yield & 13,942 & 14,900 & 13,476 & 14,679 & 17,161 \\
\hline \multicolumn{6}{|l|}{ Peanuts } \\
\hline Arca & 30,300 & 30,988 & 29,330 & 30,254 & 29,059 \\
\hline Production & 29,805 & 28,382 & 22,045 & 27,601 & 26,630 \\
\hline Yield & 984 & 916 & 752 & 912 & 916 \\
\hline \multicolumn{6}{|l|}{ Beans } \\
\hline Arca & 57,160 & 57,584 & 54,504 & 62,505 & 65,970 \\
\hline Production & 40,004 & 43,098 & 36,624 & 52,708 & 53,961 \\
\hline Yield & 700 & 748 & 672 & 843 & 818 \\
\hline \multicolumn{6}{|l|}{ Soybeans } \\
\hline Area & $1,086,043$ & $1,165,748$ & $1,176,460$ & $1,350,000$ & $1,445,365$ \\
\hline Production & $2,855,742$ & $3,053,005$ & $2,980,058$ & $3,511,049$ & $3,300,000$ \\
\hline Yield & 2,629 & 2,619 & 2,533 & 2,601 & 2,283 \\
\hline \multicolumn{6}{|l|}{ Tobaceo } \\
\hline Arca & 7,800 & (6,9] & 3,235 & 6,983 & 5,347 \\
\hline Production & 13,700 & 10,920 & 4,486 & 12,508 & 9.776 \\
\hline Yield & 1,756 & 1,580 & 1,387 & 1,791 & 1,828 \\
\hline \multicolumn{6}{|l|}{ Wheat } \\
\hline Area & 200,700 & 187,900 & 127,680 & 159,342 & 245,410 \\
\hline Produetion & 229,173 & 180,088 & 231,119 & 220,055 & 359,236 \\
\hline Yicld & $1, \downarrow 42$ & 958 & 1,810 & $!, 381$ & $1,46=$ \\
\hline
\end{tabular}

Source: Central Bank of Paraguay

1/ Agricultural years; for example, 1991 refers to the 1990/91 agricultural year. 
Table 8. Paraguay: Value-Added in Manufacturing

\begin{tabular}{|c|c|c|c|c|}
\hline & 1998 & 1999 & 2000 & $\begin{array}{l}\text { Pral. } \\
2001 \\
\end{array}$ \\
\hline \multicolumn{5}{|c|}{ (In millions of 1982 guaranies) } \\
\hline Total Manufacturing & 159,037 & 159,078 & 160,740 & 162,830 \\
\hline Consumer goods & 128,105 & 129,004 & 133,085 & 134,313 \\
\hline Handicrafts & 10,254 & 10,257 & 10,364 & 10,440 \\
\hline Foodstuff & 56,848 & 59,017 & 61,355 & 61,056 \\
\hline Diverse foodstuff & 2,243 & 2,485 & 1,984 & 2,375 \\
\hline Beverages & 16,934 & 17,180 & 17,144 & 18,589 \\
\hline Tobaceo & 866 & 763 & 702 & 714 \\
\hline Textiles & 6,550 & 5,689 & 7,603 & 8,412 \\
\hline Clothing & 120 & 120 & 121 & 121 \\
\hline Hides and furs & 7,016 & 7,244 & $7,5 ! 1$ & 8,249 \\
\hline Shoes & 2,147 & 2,044 & 1,442 & 1,100 \\
\hline Wood products & 23,517 & 22,559 & 23,208 & 21,695 \\
\hline Fumiture & 1,610 & 1,646 & 1,651 & 1,562 \\
\hline Intermediate goods & 28,018 & 27,162 & 24,722 & 25,587 \\
\hline Paper & 54 & 54 & 54 & 53 \\
\hline Print & 9,169 & 9,004 & 7,506 & 8,387 \\
\hline Industrial chemicals & 1,563 & 1,562 & 1,573 & 1,573 \\
\hline Other chemicals & 1,563 & 1,680 & 1,686 & 1,690 \\
\hline Petroleum derivatives & 3,773 & 3,312 & 2,895 & 3,208 \\
\hline Rubber products & 61 & 61 & 61 & 61 \\
\hline Flastics & 4,405 & 4,401 & 4,433 & 4,428 \\
\hline Clay and porcelane & 8 & 8 & 8 & 8 \\
\hline Glass & 176 & 185 & 186 & 157 \\
\hline Nonmetallic minerals & 6,576 & 6,250 & 5,666 & 5,391 \\
\hline Iron and steel & 262 & 237 & 243 & 221 \\
\hline Nonferrous metals & 408 & 408 & 411 & 410 \\
\hline Capital goods & 2,914 & 2,912 & 2,933 & 2,930 \\
\hline Metal products & 996 & 995 & 1,002 & 1,001 \\
\hline Machinery & 174 & 174 & 175 & 175 \\
\hline Electronics & 81 & 81 & 82 & 82 \\
\hline Transport equipment & 1,123 & 1,122 & 1,130 & 1,129 \\
\hline Scientific equipment & 3 & 3 & 3 & 3 \\
\hline Other industries & 537 & 537 & 541 & 540 \\
\hline \multicolumn{5}{|c|}{ (Percentage change) } \\
\hline Total manufacturing & 1.0 & 0.0 & 1.0 & 1.3 \\
\hline Consumer goods & 1.5 & 0.7 & 3.2 & 0.9 \\
\hline Intermediate goods & -1.4 & -3.1 & -9.0 & 3.5 \\
\hline Capital goods & 0.4 & -0.1 & 0.7 & -0.1 \\
\hline \multicolumn{5}{|c|}{ (In percent of total) } \\
\hline Total manufacturing & 100.0 & 100,0 & 100.0 & 100.0 \\
\hline Consumer goods & 80.6 & 81.1 & 82.8 & 82.5 \\
\hline Intermediate goods & 17.6 & 17.1 & 15.4 & 15.7 \\
\hline Capital goods & 1.8 & 1.8 & 1.8 & 1.8 \\
\hline
\end{tabular}

Source; Central Bank of Paraguay. 
Table 9. Paraguay: Prices

\begin{tabular}{|c|c|c|c|c|c|}
\hline & 1998 & 1999 & 2000 & 2001 & 2002 \\
\hline \multicolumn{6}{|c|}{ (In annual percentage change) } \\
\hline GDP deflator $\mathrm{V}$ & 12.4 & 2.5 & 11.9 & 1.7 & $\ldots$ \\
\hline Consumption & 6.9 & 7.5 & 12.2 & 1.0 & $\cdots$ \\
\hline Investment & 14.3 & 7.7 & 5.4 & 14.5 & $\ldots$ \\
\hline Exports & 42.1 & 6.9 & 34.4 & 22.2 & $\ldots$ \\
\hline Imports & 26.0 & 11.2 & 21.9 & 22.2 & $\ldots$ \\
\hline Consumer price index (avg) & 11.6 & 6.8 & 9.0 & 7.3 & 10.5 \\
\hline Consumer price index (cop) & 14.6 & 5.4 & 8.6 & 8.4 & 14.6 \\
\hline Tradables (eop) & 15.2 & 6.0 & 7.9 & 8.5 & 17.4 \\
\hline Nontradables (eop) & 13.8 & 4.6 & 9.7 & 8.2 & 10.4 \\
\hline Producer price index (avg) & 14.9 & 5.3 & 14.7 & 5.8 & 19.9 \\
\hline Producer price index (eop) & 18.1 & 6.8 & 10.4 & 8.6 & 36.8 \\
\hline Domestic goods (eop) & 20.4 & 2.0 & 10.0 & 2.4 & 34.1 \\
\hline Imported goods (eop) & 13.0 & 18.2 & 11.2 & 21.4 & 41.4 \\
\hline Nominal effective exchange rate (avg) & -17.1 & 2.1 & -8.1 & -5.3 & -6.5 \\
\hline Nominal cffective exchange ratc (cop) & -17.3 & 0.5 & -1.1 & -16.9 & -8.8 \\
\hline US\$/Guarani (eop) & -18.0 & -14.3 & -6.5 & -23.5 & -33.8 \\
\hline Argentine peso/Guarani (eop) & -18.0 & -14.3 & -6.5 & -23.5 & 120.6 \\
\hline Brazilian Real/Guarani (cop) & -11.2 & 26.8 & 2.2 & -9.2 & 0.8 \\
\hline Guarani/US\$ (eop) & 21.9 & 16.7 & 6.9 & 30.7 & 51.0 \\
\hline Real effective cxchange rate (avg) & -9.3 & 6.9 & -3.0 & -1.6 & -3.4 \\
\hline Real effective exchange rate (eop) & -6.4 & 2.4 & 4.3 & -12.6 & -2.6 \\
\hline RER vis-à-vis US (eop) & -7.4 & -12.1 & -1.7 & -18.4 & -25.9 \\
\hline RER vis-à-vis Argentina (eop) & -6.6 & -8.0 & 2.3 & -15.8 & 78.9 \\
\hline RER vis-à-vis Brazil (eop) & 0.2 & 22.7 & 4.7 & -8.6 & 2.7 \\
\hline \multicolumn{6}{|l|}{ Memorandum items: } \\
\hline Guarani/US\$ (eop) & 2,840 & 3,315 & 3,545 & 4,635 & 7,000 \\
\hline Guarani/US\$ (avg) & 2,706 & 3,113 & 3,481 & 4,100 & 5,679 \\
\hline
\end{tabular}

Sources: Central Bank of Paraguay; and Fund staff cstimates.

1/2001 GDP deflator numbers are preliminary. 
Table 10. Paraguay: Labor Market Indicators

(Annual percentage change)

\begin{tabular}{|c|c|c|c|c|c|}
\hline & 1998 & 1999 & 2000 & 2001 & 2002 \\
\hline \multicolumn{6}{|c|}{ I. Wages } \\
\hline Nominal wages & 10.1 & 2.2 & 11.3 & 9.1 & $\ldots$ \\
\hline Real wages & -4.0 & $-3,0$ & 2.5 & 0.7 & $\ldots$ \\
\hline Nominal minimum wage & 12.0 & 0.0 & 15.0 & 15.0 & 12.0 \\
\hline Real minimum wage & -2.3 & -5.1 & 5.9 & 6.1 & -2.3 \\
\hline \multicolumn{6}{|c|}{ II. Employment and Poverty Indicators } \\
\hline Population growth & 2.6 & 2.7 & 2.6 & 2.5 & $\ldots$ \\
\hline Unemployment rate $1 /$ & 14.3 & 15.9 & $\ldots$ & 15.3 & $\ldots$ \\
\hline Urban & 13.9 & 16.6 & $\ldots$ & 17.0 & $\ldots$ \\
\hline Rural & 14.9 & 15.0 & $\ldots$ & 13.2 & $\ldots$ \\
\hline Poverty rate 1 & 32.1 & 33.7 & $\ldots$ & 33.8 & $\ldots$ \\
\hline Urban & 23.1 & 26.7 & $\ldots$ & 27.6 & $\ldots$ \\
\hline Rural & 42.5 & 42.0 & $\ldots$ & 41.2 & $\ldots$ \\
\hline Extreme poverty rate $1 /$ & 17.3 & 15.5 & $\ldots$ & 15.4 & $\ldots$ \\
\hline Urban & 7.3 & 6.1 & $\ldots$ & 7.1 & $\ldots$ \\
\hline Rural & 28.9 & 26.5 & $\ldots$ & 25.1 & $\ldots$ \\
\hline
\end{tabular}

Sources: Technical Planning Secretariat; Central Bank of Paraguay; and Fund staff estimates.

1/ Figures correspond to the years of the household surveys, 1997/98, 1999, and 2000/01 
Table 11. Paraguay: Operations of the Consolidated Public Sector

\begin{tabular}{|c|c|c|c|c|}
\hline & 1998 & 1999 & 2000 & 2001 \\
\hline \multicolumn{5}{|c|}{ (In billions of guaranies) } \\
\hline Revenue & $5,862.0$ & $6,340,0$ & $6,122.3$ & $6,943.6$ \\
\hline Tax revenue & $2,729.1$ & $2,685.7$ & $2,980.9$ & $3,168.0$ \\
\hline Nontax revenue and grants. & $2,055.9$ & $2,190.4$ & $2,087.8$ & $2,830.5$ \\
\hline Public enterprises' operating surplus & 902.3 & $1,246.1$ & 718.0 & 712.3 \\
\hline Capital revenue & 174.7 & 217.9 & 335.6 & 232.8 \\
\hline Current expenditure & $4,094.3$ & $4,399.7$ & $5,339.8$ & $5,515.5$ \\
\hline Wages and salaries & $2,298.1$ & $2,488.2$ & $2,919.6$ & $2,965.5$ \\
\hline Goods and services & 547.3 & 434,3 & 666.9 & 557.2 \\
\hline In lurest payments & 302.2 & 321.8 & 406.5 & 496.4 \\
\hline Transfers & 822.5 & $1,063.8$ & $1,284.6$ & $1,441.6$ \\
\hline Other & 124.1 & 91.5 & 62.3 & 54.8 \\
\hline Capital expenditure and net Jending & $1,715.5$ & $2,706.8$ & $1,881.3$ & $1,691.2$ \\
\hline Current account balance & $1,593.1$ & $1,722.5$ & 446.9 & $1,195.3$ \\
\hline Orerall balance & 52.2 & -766.5 & $-1,098.8$ & -263.1 \\
\hline Financing & -678.1 & 950.6 & 949.2 & $1,059.0$ \\
\hline External debt (increase + ) & 36.9 & $1,036.6$ & $1,351.5$ & 354.1 \\
\hline Internal debt (increase + ) & -870.4 & -264.4 & -312.0 & 528.0 \\
\hline Monetary expansion & 155.4 & 178.4 & -48.0 & 85.0 \\
\hline Float and errors & 625.9 & -184.2 & -42.3 & 91.9 \\
\hline \multicolumn{5}{|c|}{ (In percent of GDP) } \\
\hline Revenue & 25.0 & 26.3 & 22.7 & 24.7 \\
\hline Tax revenue & 11.6 & 11.1 & 11.1 & 11.3 \\
\hline Nontax revenue and grants & 8.8 & 9.1 & 7.8 & 10.1 \\
\hline Public enterprises' operating surplus & 3.8 & 5.2 & 2.7 & 2.5 \\
\hline Capital revenue & 0.7 & 0.9 & 1.2 & 0.8 \\
\hline Current expenditure & 17.5 & 18.2 & 19.8 & 19.6 \\
\hline Wages and salaries & 9.8 & 10.3 & 10.8 & 10.5 \\
\hline Goods and services & 2.3 & 1.8 & 2.5 & 2.0 \\
\hline Interest payments & 1.3 & 1.3 & 1.5 & 1.8 \\
\hline Translors & 3.5 & 4,4 & 4.8 & 5.1 \\
\hline Other & 0.5 & 0.4 & 0.2 & 0.2 \\
\hline Capital expenditure and net lending & 7.3 & 11.2 & 7.0 & 6.0 \\
\hline Capital expenditure & 7.7 & 11.5 & 6.9 & 5.9 \\
\hline Net lending by the centrol bank & -0.4 & -0.3 & 0.1 & 0.1 \\
\hline Current account balance & 6.8 & 7.1 & 1.7 & 4.3 \\
\hline Overall balance & 0.2 & -3.2 & -4.1 & -0.9 \\
\hline Financing & -2.9 & 3.9 & 3.5 & 3.8 \\
\hline External debt (increase + ) & 0.2 & 4.3 & 5.0 & 1.3 \\
\hline Intertial debt (increase + ) & -3.7 & -1.1 & -1.2 & 1.9 \\
\hline Monetary expansion & 0.7 & 0.6 & -0.9 & 0.0 \\
\hline Float and errors & 2.7 & 0.0 & 0.0 & 0.0 \\
\hline
\end{tabular}

Sources: Ministry of Finance; and Fund Staff estimates. 
Table 12. Paraguay: Operations of the Central Government

\begin{tabular}{|c|c|c|c|c|c|}
\hline & 1.998 & 1999 & 2000 & 2001 & $\begin{array}{l}\text { Pret. } \\
2002\end{array}$ \\
\hline \multicolumn{6}{|c|}{ (In bilkions of guatiaries) } \\
\hline Total revenue & $3,703.6$ & $3,989.2$ & $4,214,0$ & $4,891,5$ & $5,048,3$ \\
\hline Tax revenue & $2,729.1$ & $2,685.7$ & $2,977.2$ & $3,164.8$ & $3,233.0$ \\
\hline Social security contributions & 244.6 & 288.0 & 300.5 & 313.4 & 309.6 \\
\hline Income taxes & 474.4 & 552.2 & 479.1 & $464 . I$ & 450.5 \\
\hline Taxes on gaods and services & $1,483,5$ & $1,446.7$ & $1,710.1$ & $1,888.8$ & $1,965.7$ \\
\hline Excise taxes & 302.7 & 308,2 & 435.3 & 573.6 & 608.7 \\
\hline Valne added tax & $1,063.7$ & $3,048.4$ & $1,150,3$ & $1,204.3$ & $1,235.4$ \\
\hline Starmp tax & 76.1 & 68.8 & 69.9 & 79.5 & $\$ 6.5$ \\
\hline Othet & 41.0 & 21.3 & 54.6 & 31.4 & 35.1 \\
\hline Taxes on intanetional transactions & 643.7 & 488.9 & 612.]^{\circ}$ & 609.4 & 628.8 \\
\hline Import duties & 526.6 & 398.8 & $4 \$ 7.6$ & 498,5 & 507.2 \\
\hline Other & 117.1 & 90.1 & 124.5 & 110.9 & 121.6 \\
\hline Nontax revenuse & 937.1 & $1,260.1$ & $1,152.5$ & $1,669.4$ & $1,308.6$ \\
\hline Itaipu and Yacurela & 658.0 & 892.2 & 664.8 & $1,146.1$ & $1,259.2$ \\
\hline Other (including Erants) & 279.1 & 367.9 & 487.7 & 523.3 & 549.4 \\
\hline Capital revenue & 37.4 & 43.5 & 84.3 & 57.3 & 6.7 \\
\hline Current expenditurts & $3,199.7$ & $3,549.5$ & $4,368,9$ & $4,378,2$ & $4,773.7$ \\
\hline Wages and salaries & $1,818.0$ & $2,021.4$ & $2,400,9$ & $2,400.1$ & $2,581.9$ \\
\hline Goods and services & 332.2 & 250.4 & 420.0 & 295.4 & 353.0 \\
\hline Interest payments & 170.4 & 193.2 & 312.5 & 377.5 & $4 \$ 6.4$ \\
\hline Transfers & 774.9 & $1,009.5$ & $1,196.2$ & $1,270.0$ & $1,326.3$ \\
\hline Other & 104.1 & 75.1 & 39.4 & 34.8 & 56.2 \\
\hline Capital expendi tures & 730.9 & $1,304.9$ & 994.1 & 831.5 & $1,299.2$ \\
\hline Capital formation & 664.5 & 7663 & 747.7 & 794.4 & $1,147,2$ \\
\hline Capital transfers & 94.3 & 430.4 & 149.1 & 115.9 & 139.9 \\
\hline Other & -27.9 & 108.2 & 91.3 & .78 .8 & 12.2 \\
\hline Current account balance & 466.5 & 396.2 & -239.2 & 456.0 & 267.9 \\
\hline Overall balance & -227.1 & -865.2 & $-1,148,9$ & -318.1 & $-1,024,6$ \\
\hline Financing & 227.1 & 865.2 & $1,168.9$ & 318.1 & $1,024.6$ \\
\hline External debt (increase +) & 108.3 & $1,478.0$ & 465.2 & .29 .1 & 314.9 \\
\hline Internal debt (increase +) & 256.4 & -67.7 & -130.4 & 528.2 & 122.1 \\
\hline Central bank deposits (increase -) & -520.3 & -716.5 & 514,8 & 97.2 & 10.0 \\
\hline Float and exrors & 382.7 & 171.4 & 299.4 & -278.1 & 577.7 \\
\hline \multicolumn{6}{|c|}{ (In percent of GDP) } \\
\hline Total revenue & 15.8 & 16.5 & 15.7 & 17.4 & 16.1 \\
\hline Tax revenue & 11.6 & 11.1 & 11.1 & 11,3 & 10.3 \\
\hline Social security contributions & 1.0 & 1.2 & 1.1 & 1.1 & 1.0 \\
\hline Income taxes & 2.0 & 2.3 & 1.8 & 1.7 & 1.4 \\
\hline Taxes on Boods and services & 6.3 & 6.0 & 6.4 & 6.7 & 6.3 \\
\hline Taxes on internationg] transactions & 2.7 & 2.0 & 2.3 & 2.2 & 2.0 \\
\hline Nontax revenue & 4.0 & 5.2 & 4.3 & 5.9 & 5.8 \\
\hline Itaipu & 2.8 & 3.7 & 2.5 & 4.1 & 4.0 \\
\hline Otter (including grants) & 1.2 & 1.5 & 1.8 & 1.9 & 1.8 \\
\hline Capital revenue & 0.2 & 0.2 & 0.3 & 0.2 & 0.0 \\
\hline Current expenditures & 13.7 & 14.7 & 16.2 & 15.6 & 15.2 \\
\hline Wages and salaries & 7.8 & 8.4 & 8.9 & 8.5 & 8.2 \\
\hline Goods and services & 1.4 & 1.0 & 1.6 & 1.1 & 1.1 \\
\hline Intercst payments & 0.7 & 0.8 & 1.2 & 1.3 & 1.5 \\
\hline Transfers & 3.3 & 4.2 & 4.4 & 4.5 & 4.2 \\
\hline Other & 0.4 & 0.3 & 0.1 & 0.1 & 0.2 \\
\hline Capital expenditures & 3,1 & 5.4 & 3.7 & 3.0 & 4.1 \\
\hline Current account balance & 2.0 & 1.6 & -0.9 & 1.6 & 0.9 \\
\hline Dverall balance & -1.0 & -3.6 & -4.3 & -1.1 & -3.3 \\
\hline Financing & 1.0 & 3.6 & 4.3 & 1.1 & 3.3 \\
\hline External debt (increase +) & 0.5 & 6.1 & 1.7 & -0.1 & 1.0 \\
\hline Internal debt (increase +) & 1.1 & .0 .3 & -0.5 & 1.9 & 0.4 \\
\hline Central bank deposits (increase -) & -2.2 & .3 .0 & 19 & 0.3 & 0.0 \\
\hline Floal and errors & 1.6 & 0.7 & 1.1 & -1.0 & 1,8 \\
\hline
\end{tabular}

Soutces: Ministry of Finance; and Fund staff estimates. 
Table 13. Paraguay: Operations of the Social Security Institute (IPS)

\begin{tabular}{|c|c|c|c|c|}
\hline & 1998 & 1999 & 2000 & 2001 \\
\hline \multicolumn{5}{|c|}{ (In billions of guaranies) } \\
\hline Revenues & 745.9 & 505.1 & 580.8 & 766.8 \\
\hline Social security contributions & 536.1 & 451.5 & 501.8 & 622.9 \\
\hline Other current revenue & 198.5 & 44.6 & 60.0 & 108.4 \\
\hline Capital revenue & 11.3 & 9.0 & 19.1 & 35.5 \\
\hline Current expenditures & 501.0 & 394.7 & 498.3 & 622.0 \\
\hline Wages and salaries & 200.5 & 148.1 & 180.0 & 229.1 \\
\hline Goods and services & 134.6 & 98.0 & 144.1 & 159.8 \\
\hline Interest payments & 0.0 & 0.0 & 0.0 & 0.0 \\
\hline Transfers & 150.5 & 140.5 & 159.9 & 217.1 \\
\hline Other & 15.4 & 8.1 & 14.4 & 16.1 \\
\hline Capital expenditures & 48.6 & 36.7 & 34.2 & 46.8 \\
\hline Current account balance & 233.6 & 101.4 & 63.4 & 109.3 \\
\hline Overall balance & 196.3 & 73.7 & 48.3 & 98.0 \\
\hline Financing & -196.3 & -73.7 & -48.3 & -98.0 \\
\hline External debt (increase + ) & 0.0 & 0.0 & 0.0 & 0.0 \\
\hline Internal debt (increase +) & -196.3 & -73.7 & -48.3 & -98.0 \\
\hline Central bank deposits (+ decrease) & 0.0 & 0.0 & 0.0 & 0.0 \\
\hline Float and errors & 0.0 & 0.0 & 0.0 & 0.0 \\
\hline \multicolumn{5}{|c|}{ (In pencent of GDP) } \\
\hline Revenues & 3.2 & 2.1 & 2.2 & 2.7 \\
\hline Current expenditure & 2.1 & 1.6 & 1.9 & 2.2 \\
\hline Wages and salaries & 0.9 & 0.6 & 0.7 & 0.8 \\
\hline Goods and services & 0.6 & 0.4 & 0.5 & 0.6 \\
\hline Interest payments & 0.0 & 0.0 & 0.0 & 0.0 \\
\hline Transfers & 0.6 & 0.6 & 0.6 & 0.8 \\
\hline Other & 0.1 & 0.0 & 0.1 & 0.1 \\
\hline Capital expenditare & 0.2 & 0.2 & 0.1 & 0.2 \\
\hline Current account balance & 1.0 & 0.4 & 0.2 & 0.4 \\
\hline Overall balance & 0.8 & 0.3 & 0.2 & 0.3 \\
\hline Financing & -0.8 & -0.3 & -0.2 & $-\mathbf{0 . 3}$ \\
\hline Extertal debt (increase + ) & 0.0 & 0.0 & 0.0 & 0.0 \\
\hline Internal debt (increase +) & -0.8 & -0.3 & -0.2 & -0.3 \\
\hline Central bank dcposits (increase -) & 0.0 & 0.0 & 0.0 & 0.0 \\
\hline Float and errors & 0.0 & 0.0 & 0.0 & 0.0 \\
\hline
\end{tabular}

Sources: The Ministry of Finance; and Fund staff estimates. 
Table 14. Paraguay: Rest of General Government 1/

(In billions of guaranies)

\begin{tabular}{lrrrr}
\hline & 1998 & 1999 & 2000 & 2001 \\
\hline Revenues & & & & \\
Current transfers from central government & $\mathbf{3 7 1 . 6}$ & $\mathbf{4 7 8 . 7}$ & $\mathbf{5 1 6 . 5}$ & $\mathbf{3 6 2 . 3}$ \\
Other current revenues & 196.1 & 227.2 & 238.9 & 214.4 \\
Capital revenues & 112.5 & 132.9 & 131.1 & 121.3 \\
Current expenditures & 63.1 & 118.6 & 146.5 & 26.6 \\
Wages and salaries & $\mathbf{2 7 3 . 7}$ & $\mathbf{2 9 8 . 6}$ & $\mathbf{4 0 0 . 4}$ & $\mathbf{3 1 5 . 2}$ \\
Goods and services & $\mathbf{1 8 0 . 0}$ & 201.9 & 213.0 & 210.2 \\
Interest payments & 48.0 & 54.6 & 68.8 & 61.9 \\
Transfcrs & 0.2 & 0.2 & 0.1 & 0.4 \\
Other & 43.7 & 40.2 & $\mathbf{1 1 7 . 4}$ & 40.9 \\
Capital expenditures & 1.8 & 1.7 & 1.2 & 1.7 \\
Current account balance & $\mathbf{9 9 . 2}$ & $\mathbf{1 0 0 . 8}$ & $\mathbf{1 2 4 . 9}$ & $\mathbf{4 8 . 4}$ \\
Overall balance & $\mathbf{3 4 . 9}$ & $\mathbf{6 1 . 5}$ & $\mathbf{- 3 0 . 4}$ & $\mathbf{2 0 . 5}$ \\
Financing 1/ & $\mathbf{- 1 . 2}$ & $\mathbf{7 9 . 3}$ & $\mathbf{- 8 . 8}$ & $\mathbf{- 1 . 3}$ \\
External debt (increase +) & $\mathbf{1 . 2}$ & -79.3 & $\mathbf{8 . 8}$ & $\mathbf{1 . 3}$ \\
Internal debt (increase +) & 34.8 & 0.0 & 0.0 & -0.5 \\
Central bank deposits (increase -) & -33.6 & -79.3 & 8.8 & 1.8 \\
Float and errors & 0.0 & -0.3 & $\mathbf{- 0 . 3}$ & 0.0 \\
\hline
\end{tabular}

Source: Ministry of Finance.

1/ Includes local governments, development and regulatory institutions, and educational institutions (UNA, UNE, UNI, UNP) 
Table 15. Paraguay: Operations of the Public Enterprises

\begin{tabular}{|c|c|c|c|c|}
\hline & 1998 & 1999 & 2000 & 2001 \\
\hline \multicolumn{5}{|c|}{ (In billions of guaranies) } \\
\hline Revenues & $2,287.2$ & $2,407.5$ & $3,007.4$ & $3,461.0$ \\
\hline Sales of goods and services & $2,279.0$ & $2,392.5$ & $2,750.8$ & $3,433.4$ \\
\hline Current transfers from central government & 6.9 & 8.9 & 7.1 & 12.6 \\
\hline Capital revenue & 1.3 & 6.0 & 0.5 & 3.0 \\
\hline Current expenditures & $1,396.2$ & $1,197.4$ & $2,345.8$ & $\mathbf{2 , 7 7 0 . 2}$ \\
\hline Wages and salaries & 457.5 & 479.0 & 479.8 & 492.2 \\
\hline Goods and services & 720.8 & 357.2 & $1,584.1$ & $1,903.4$ \\
\hline Interest payments & 27.6 & 55.0 & 52.8 & 56.2 \\
\hline Transfers & 34.5 & 65.7 & 86.6 & 97.6 \\
\hline Other & 155.7 & 240.4 & 142.5 & 220.8 \\
\hline Capital expenditures & 803.8 & $1,272.1$ & 582.8 & 618.1 \\
\hline Current account balance & 889.7 & $1,204.0$ & 661.0 & 687.8 \\
\hline Overall balance & 87.2 & -62.1 & 78.7 & 72.7 \\
\hline Financing & -87.2 & 62.1 & -78.7 & -72.7 \\
\hline External debt (increase + ) & 49.3 & 49.9 & 84.8 & 49.5 \\
\hline Internal debt (increase + ) & 23.7 & 12.0 & -163.0 & -122.0 \\
\hline Central bank deposits (increase -) & 8.2 & 36.6 & 15.4 & 53.7 \\
\hline Float and errors & -168.4 & -36.4 & -15.9 & -53.9 \\
\hline \multicolumn{5}{|c|}{ (In percent of GDP) } \\
\hline Revenues & 9.8 & 10.0 & 11.2 & 12.3 \\
\hline Current expenditures & 6.0 & 5.0 & 8.7 & 9.9 \\
\hline Wages and salaries & 2.0 & 2.0 & 1.8 & 1.8 \\
\hline Goods and services & 3.1 & 1.5 & 5.9 & 6.8 \\
\hline Interest payments & 0.1 & 0.2 & 0.2 & 0.2 \\
\hline Transfers & 0.1 & 0.3 & 0.3 & 0.3 \\
\hline Other & 0.7 & 1.0 & 0.5 & 0.8 \\
\hline Capital expenditures & 3.4 & 5.3 & 2.2 & 2.2 \\
\hline Current account balance & 3.8 & 5.0 & 2.5 & 2.4 \\
\hline Overall balance & 0.4 & -0.3 & 0.3 & 0.3 \\
\hline Financing & -0.4 & 0.3 & $-\mathbf{0 . 3}$ & -0.3 \\
\hline External debt (increase +) & 0.2 & 0.2 & 0.3 & 0.2 \\
\hline Internal debt (increase +) & 0.1 & 0.0 & -0.6 & -0.4 \\
\hline Central bank deposits (increase -) & 0.0 & 0.2 & 0.1 & 0.2 \\
\hline Float and errors & -0.7 & -0.2 & -0.1 & -0.2 \\
\hline
\end{tabular}

Sources: Ministry of Finance; and Fund staff estimates. 
Table 16. Paraguay: Quasi-Fiscal Operations of the BCP

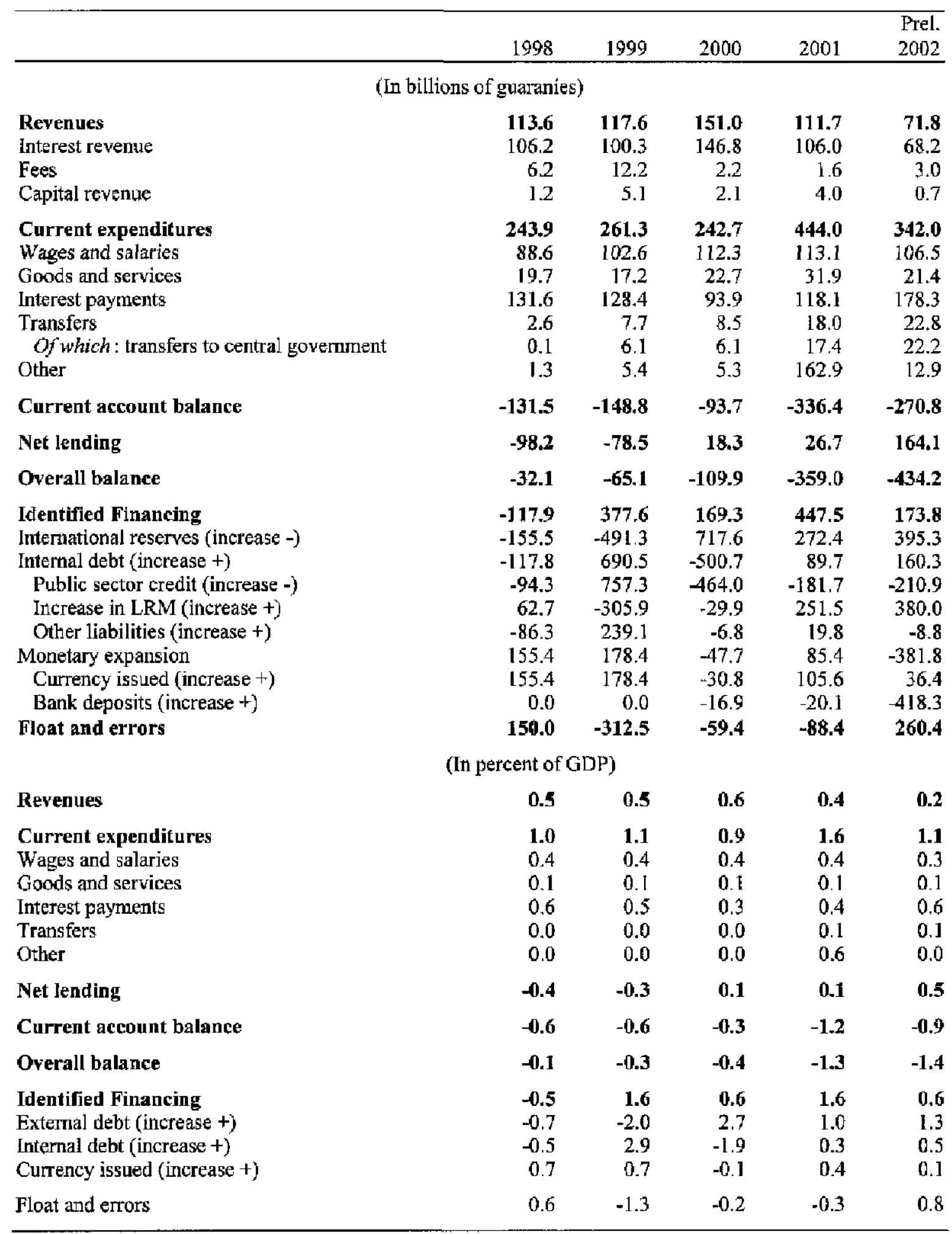

Sources: Central Bank of Paraguay; and Fund staff estimates. 
Table 17. Paraguay: Central Bank Balance Sheet

(In billion of guaranies, end of period)

\begin{tabular}{|c|c|c|c|c|c|}
\hline & 1998 & 1999 & 2000 & 2001 & 2002 \\
\hline Net international reserves & 2,484 & 3,276 & 2,736 & $\mathbf{3 , 3 5 2}$ & 4,489 \\
\hline (In millions of U.S. dollars) & 875 & 988 & 772 & 723 & 641 \\
\hline Net domestic assets & $-1,075$ & $-1,688$ & $-1,180$ & $-1,689$ & $-2,790$ \\
\hline Net nonfinancial public sector & 612 & 61 & 534 & 872 & 1,581 \\
\hline Net credit to the general government & 336 & -255 & 200 & 698 & 1,354 \\
\hline Central Government & 334 & -257 & 199 & 697 & 1,354 \\
\hline Credit & 880 & 1,062 & 1,003 & 1,403 & 2,050 \\
\hline Deposits & 546 & 1,319 & 804 & 707 & 697 \\
\hline Rest of general government & 2 & 2 & 1 & 1 & 1 \\
\hline Credit & 2 & 2 & 1 & 1 & 1 \\
\hline Deposits & 0 & 0 & 0 & 0 & 0 \\
\hline Net credit to public enterprises & 277 & 316 & 334 & 174 & 227 \\
\hline Credit & 306 & 331 & 349 & 177 & 230 \\
\hline Deposits & 29 & 15 & 15 & 3 & 3 \\
\hline Net credit to the financial system & -238 & -346 & -376 & -672 & -637 \\
\hline Credit & 1,178 & 1,243 & 1,256 & 1,282 & 1,455 \\
\hline Deposits & 1,416 & 1,589 & 1,632 & 1,954 & 2,092 \\
\hline Net credit to the banking system & $-1,229$ & $-1,485$ & $-1,533$ & $-1,827$ & $-1,969$ \\
\hline Total credit to banks & 45 & 5 & 7 & 7 & 4 \\
\hline Loans and liquidity ( + active call) & 0 & 0 & 0 & 0 & 0 \\
\hline Other loans to banks & 45 & 5 & 7 & 7 & 4 \\
\hline Deposits and reserve requirements & 1,275 & 1,489 & 1,541 & 1,833 & 1,973 \\
\hline Net credit to the rest of financial entities & 991 & 1,139 & 1,157 & 1,155 & 1,332 \\
\hline Credit & 1,132 & 1,238 & 1,248 & 1,276 & 1,451 \\
\hline Deposits & 141 & 99 & 91 & 121 & 119 \\
\hline Net credit to the private sector & -40 & -50 & -55 & -59 & -92 \\
\hline Credit & 18 & 22 & 26 & 28 & 32 \\
\hline Deposits and liabilities & 0 & 0 & 0 & 0 & 0 \\
\hline Restricted deposits & 58 & 72 & 80 & 87 & 124 \\
\hline Open market operations (LRM and passive call) & 382 & 76 & 46 & 297 & 677 \\
\hline Medium- and long-term external liabilities & 171 & 176 & 155 & 331 & 478 \\
\hline Capital and reserves & 863 & 1,209 & 1,187 & 1,556 & 1,870 \\
\hline Provisions & 756 & 929 & 964 & 1,033 & 1,056 \\
\hline Other assets net & 763 & 1,037 & 1,069 & 1,388 & 438 \\
\hline Currency issue & 1,409 & 1,587 & $\mathbf{1 , 5 5 7}$ & $\mathbf{1}, 662$ & 1,699 \\
\hline Growth & 12.4 & 12.7 & -1.9 & 6.8 & 2.2 \\
\hline
\end{tabular}

Sources: Central Bank of Paraguay; and Fund staff estimates. 
Table 18. Paraguay: Commercial Banks Balance Sheet

(In billion or guatanies)

\begin{tabular}{|c|c|c|c|c|c|}
\hline \multirow{3}{*}{ Net international assets } & \multicolumn{5}{|c|}{ December } \\
\hline & 1998 & 1999 & \multirow{2}{*}{$\frac{2000}{1,497.6}$} & \multirow{2}{*}{$\begin{array}{l}2001 \\
1,744.5\end{array}$} & \multirow{2}{*}{$\frac{2002}{1,638.1}$} \\
\hline & 566.7 & 983.8 & & & \\
\hline (In millions of U.S. dollars) & 199.5 & 296.8 & 422.5 & 376.4 & 234.0 \\
\hline Gross external assets & $1,178.5$ & $1,327.0$ & $1,759.7$ & $2,068.2$ & $2,015,0$ \\
\hline International short -run liabilities & 611.8 & 343.2 & 262.1 & 323.8 & 376.8 \\
\hline Net domestic assets & $4,507.4$ & $5,061.3$ & $4,916.5$ & $5,936.3$ & $6,148.1$ \\
\hline Net public sector & -530.5 & .403 .6 & -646.5 & -730.9 & -594.6 \\
\hline Net credit to the general government & -407.1 & -276.5 & -425.3 & -618.9 & -428.4 \\
\hline Central government & 136.6 & 325.3 & 262.6 & 203.0 & 436.7 \\
\hline Credit & 283.3 & 449.5 & 353.5 & 291.6 & 518.9 \\
\hline Deposits & 146.6 & 124.3 & 90.8 & 88.6 & 82.2 \\
\hline Rest of general government & -543.8 & -601.8 & -687.9 & -821.8 & -865.1 \\
\hline Credit & 36.6 & 5.5 & 9.0 & 4.3 & 1.7 \\
\hline Deposits & 580.4 & 607.3 & 696.8 & 826.2 & 866.8 \\
\hline Net credit to public enterprises & -123.4 & -127.0 & -221.2 & -112.1 & -166.2 \\
\hline Credit & 0.8 & 1.3 & 0,0 & 0.0 & 0.0 \\
\hline Deposits & 124.1 & 128.3 & 221.2 & 112.1 & 166.2 \\
\hline Net position in the $\mathrm{BCP}$ & $1,584.9$ & $1,620.5$ & $1,685.8$ & $2,174,2$ & $2,413.4$ \\
\hline Deposits in the BCP and holding of LRM & $1,618.1$ & $1,689.1$ & $1,772.3$ & $2,263.5$ & $2,544.6$ \\
\hline Deposits in the ВCP & $1,418.5$ & $1,631.2$ & $1,734.3$ & $2,100.2$ & $2,246.7$ \\
\hline Holding of LRM & 199.6 & 58.0 & 38.0 & 163.3 & 297.9 \\
\hline Liabilities to the BCP & 33.3 & 68.7 & 86.5 & 89.3 & 131.1 \\
\hline Net credit to the rest of financial entities & -4.6 & -11.5 & -18.4 & -142.9 & -185.7 \\
\hline Credit & 45,5 & 57.0 & 83.7 & 92.6 & 29.6 \\
\hline Deposits & 50.1 & 68.5 & 102.1 & 235.5 & 215.3 \\
\hline Credit to the private sector & $4,965.0$ & $5,518.8$ & $5,797.2$ & $6,605.5$ & $6,592.0$ \\
\hline Restricted deposits & 18.7 & 23.1 & 60.2 & 51.2 & 92.4 \\
\hline Medium- and long-term external liabilities & 44.4 & 51.5 & 108.9 & 121.5 & 106.1 \\
\hline Capital and reserves & $1,325.4$ & $1,267.2$ & $1,345.9$ & $1,502.0$ & $1,414,9$ \\
\hline Provisions & 284.1 & 448.3 & 544.2 & 630.4 & 957.1 \\
\hline Other assets net & 165.3 & 127.2 & 157.5 & 335.4 & 493.5 \\
\hline Private sector deposits & $5,074.1$ & $6,045.1$ & $6,414.1$ & $7,680.7$ & $7,786.2$ \\
\hline Demand deposits & 654.8 & 701.9 & $1,088.8$ & $1,243.5$ & $1,190.2$ \\
\hline Quasi-money & $1,390.3$ & $1,561.3$ & $1,326.0$ & $1,305.7$ & $1,197.9$ \\
\hline Time and savings deposits & $1,001.2$ & $1,049.7$ & 746.7 & 680.1 & 522.0 \\
\hline Certificates of deposits and notes (pagares) & 389.1 & 511.6 & 579.3 & 625.6 & 675.9 \\
\hline Foreign currency deposits & 3,0006 & $3,759.9$ & $3,946.1$ & $5,082.5$ & $5,197.7$ \\
\hline (In million of U.S. dollars) & $1,056.5$ & $1,134.2$ & $1,113.2$ & $1,096.5$ & 742.5 \\
\hline Obligaciones por intermed. financiera & 13.1 & 9.4 & 39.3 & 12,1 & 17.1 \\
\hline Trust and mutual funds & 0.0 & 0.0 & 0.0 & 0.0 & 0.0 \\
\hline Bonds and securities & 15.4 & 12.7 & 13.8 & 36.9 & 183.4 \\
\hline
\end{tabular}

Sources: Central Bank of Paraguay; and Fund staff estimates. 
Table 19. Paraguay: Monetary Survey

(In billions of guaranies, end of period)

\begin{tabular}{|c|c|c|c|c|c|}
\hline & 1998 & 1999 & 2000 & 2001 & 2002 \\
\hline Net international reserves & $\mathbf{3 , 0 5 1}$ & 4,260 & 4,234 & 5,096 & 6,127 \\
\hline (In millions of $U$.S, dollars) & 1,074 & 1,285 & 1,194 & 1,099 & 875 \\
\hline Net domestic assets & 3,469 & 3,202 & 3,515 & 4,083 & 3,391 \\
\hline Net credit to the public sector & 82 & -343 & -112 & 141 & 987 \\
\hline Net credit to the general government & -71 & -532 & -225 & 79 & 926 \\
\hline Net credit to the rest of the public sector & 153 & 189 & 113 & 62 & 61 \\
\hline Net credit to the rest of financial entities & 986 & 1,128 & 1,139 & 1,012 & 1,146 \\
\hline Credit to the private sector & 4,983 & 5,541 & 5,823 & 6,633 & 6,624 \\
\hline Restricted deposits & -77 & -95 & -140 & -138 & -217 \\
\hline Medium- and long-term external liabilities & -216 & .227 & -264 & -453 & -584 \\
\hline Capital and reserves & $-2,189$ & $-2,477$ & $-2,533$ & $-3,058$ & $-3,284$ \\
\hline Provisions & $-1,040$ & $-1,378$ & $-1,509$ & $-1,664$ & $-2,013$ \\
\hline Other assets net & 939 & 1,053 & 1,112 & 1,610 & 732 \\
\hline Liabilities with the private sector $1 /$ & 6,520 & 7,462 & 7,749 & 9,179 & $\mathbf{9 , 5 1 8}$ \\
\hline Trust and mutual funds & 0 & 0 & 0 & 0 & 0 \\
\hline M4 $=$ M3 plus bonds, LRM, liabilities & 6,520 & 7,462 & 7,749 & 9,179 & 9,518 \\
\hline Bonds and securities (banks) & 15 & 13 & 14 & 37 & 183 \\
\hline Liabilities of financial intermediation (banks) & 13 & 9 & 39 & 12 & 17 \\
\hline LRM with the private sector (BCP) & 182 & 18 & 8 & 121 & 281 \\
\hline $\mathbf{M 3}=\mathbf{M} 2$ plus foreign currency deposits & 6,310 & 7,422 & 7,688 & 9,009 & 9,037 \\
\hline Foreign currency deposits & 3,001 & 3,760 & 3,946 & 5,082 & 5,198 \\
\hline M2 $=$ M1 plus quasi-money & 3,309 & 3,662 & 3,742 & 3,926 & 3,839 \\
\hline Quasi-money & 1,390 & 1,561 & 1,326 & 1,306 & 1,198 \\
\hline Time and savings deposits & 1,001 & 1,050 & 747 & 680 & 522 \\
\hline \multicolumn{6}{|l|}{ Certificates of deposits and investment } \\
\hline titles (pagares) & 389 & 512 & 579 & 626 & 676 \\
\hline M1 (narrow money) & 1,919 & 2,101 & 2,416 & 2,621 & 2,641 \\
\hline Currency in circulation outside banks & 1,264 & 1,399 & 1,327 & 1,377 & 1,451 \\
\hline Currency issue & 1,409 & 1,587 & 1,557 & 1,662 & 1,699 \\
\hline Cash in vaults & 145 & 189 & 229 & 285 & 247 \\
\hline Demand deposits & 655 & 702 & 1,089 & 1,243 & 1,190 \\
\hline
\end{tabular}

Sources: Central Bank of Paraguay; and Fund staff estimates.

1/ Includes certral bank bills (LRMs) outside the banking system. 
Table 20. Paraguay; Interest Rate Structure

(In percent, end of period)

\begin{tabular}{lrrrrr}
\hline & \multicolumn{5}{c}{ Decernber } \\
\cline { 2 - 6 } & 1998 & 1999 & 2000 & 2001 & 2002 \\
\hline & I. Banks: Lending & & & \\
Lending rate, local currency & & & & & \\
Commercial & 31.5 & 27.8 & 25.3 & 30.6 & 36.8 \\
Development & 31.6 & 29.2 & 34.4 & 32.7 & 35.2 \\
Personal & 37.7 & 38.3 & 35.2 & 37.1 & 45.4 \\
Other & 25.0 & 25.2 & 24.4 & 20.1 & 21.3 \\
& & & & & \\
Lending rate, U.S. dollar loans & & & & & \\
Commercial & 11.6 & 11.8 & 10.9 & 9.2 & 8.9 \\
Development & 13.8 & 11.8 & 12.1 & 8.8 & 9.4 \\
Personal & 16.8 & 17.5 & 16.2 & 12.2 & 10.2
\end{tabular}

II. Banks: Deposits

\section{Deposit rates in local currency \\ Demand deposits \\ Fixed term deposits \\ Less than 90 days \\ Less than 180 days \\ Less than 365 days \\ More than 365 days \\ Certificate of deposits \\ Up to 180 days \\ Less than 365 days \\ More than 365 days}

$\begin{array}{rrrrr}4.9 & 7.1 & 5.6 & 5.8 & 9.1 \\ 23.1 & 18.4 & 13.8 & 21.5 & 24.5 \\ 24.4 & 18.5 & 12.3 & 21.9 & 24.2 \\ 21.5 & 19.6 & 14.8 & 18.6 & 24.3 \\ 18.6 & 14.8 & 13.9 & 16.4 & 16.5 \\ 19.7 & 15.5 & 19.6 & 26.2 & 30.0 \\ 21.5 & 19.8 & 20.3 & 22.7 & 23.1 \\ 22.3 & 19.7 & 19.8 & 20.5 & 21.7 \\ 21.3 & 19.4 & 20.1 & 22.1 & 22.5 \\ 20.3 & 20.4 & 21.0 & 23.8 & 25.0\end{array}$

\section{Deposit rates in foreign currency}

Demand deposits

Fixed term deposits

Less than 90 days

Less than 180 days

Less than 365 days

More than 365 days

Certificate of deposits

Up to 180 days

Less than 365 days

More than 365 days

$\begin{array}{rrrrr}2.6 & 2.6 & 2.3 & 1.8 & 0.7 \\ 4.5 & 4.9 & 4.3 & 1.6 & 1.5 \\ 4.1 & 4.7 & 4.2 & 1.7 & 1.1 \\ 4.9 & 4.9 & 4.3 & 1.6 & 1.7 \\ 4.8 & 5.1 & 4.4 & 1.4 & 1.4 \\ 6.4 & 4.5 & 5.0 & 2.7 & 2.2 \\ 6.9 & 6.7 & 6.4 & 3.5 & 3.4 \\ 6.3 & 7.4 & 5.5 & 3.5 & \ldots \\ 9.0 & 5.6 & 5.3 & 1.3 & 1.1 \\ 6.7 & 6.4 & 6.5 & 3.7 & 3.7\end{array}$

III. Other

Central bank bills

26.2

13.2

5.9

21.0

24.7

Soutce: Central Bank of Paraguay. 
Table 21. Paraguay: Banking System Indicators

\begin{tabular}{|c|c|c|c|c|c|}
\hline & 1998 & 1999 & 2000 & 2001 & 2002 \\
\hline \multicolumn{6}{|l|}{ Total system } \\
\hline Share in assets & 100.0 & 100.0 & 100.0 & 100.0 & 100.0 \\
\hline Capital adequacy ratio (percent) & 20.7 & 17.4 & 17.6 & 16.9 & 17.9 \\
\hline NPLs (percent) total loans & 13.3 & 16.5 & 16.6 & 16.5 & 19.7 \\
\hline Provisions (percent) NPLs & 30.6 & 34.9 & 38.8 & 37.0 & 46.6 \\
\hline Rate of return on assets (ROA) & 3.0 & 2.0 & 1.4 & 2.2 & 1.0 \\
\hline Rate of retum on equity (ROE) & 23.9 & 18.5 & 12.4 & 21.2 & 9.0 \\
\hline Liquid assets (percent) of total assets $1 /$ & 25.3 & 25.1 & 21.7 & 23.0 & 25.1 \\
\hline \multicolumn{6}{|l|}{ Total foreign banks } \\
\hline Share in assets & 51.9 & 52.1 & 47.0 & 45.1 & 48.4 \\
\hline Capital adequacy ratio (percent) & 18.2 & 17.0 & 17.1 & 16.0 & 17.6 \\
\hline NPLs (percent) total kans & 5.6 & 10.3 & 14.1 & 15.3 & 20.1 \\
\hline Provisions (percent) KPLs & 41.7 & 39.2 & 40.2 & 42.6. & 57.5 \\
\hline Rate of return on assets (ROA) & 5.2 & 3.1 & 2.3 & 3.1 & 1.6 \\
\hline Rate of return on equity (ROE) & 55.7 & 31.7 & 20.2 & 30.4 & 15.0 \\
\hline Liquid assets (percent) of total assets $U$ & 28.7 & 24.6 & 21.6 & 22.2 & 27.1 \\
\hline \multicolumn{6}{|l|}{ Total majority foreign banks } \\
\hline Shate in assets & 27.0 & 27.3 & 34.9 & 38.3 & 33.2 \\
\hline Capital adoquacy ratio (percant) & 21.0 & 17.7 & 17.7 & 16.8 & 19.9 \\
\hline NPLs (percent) total loans & 6.0 & 11.3 & 10.6 & 10.6 & 10.4 \\
\hline Provisions (percent) NPLs & 39.7 & 33.2 & 39.7 & 36.8 & 43.8 \\
\hline Rate of return on assets (ROA) & 4.3 & 1.2 & 1.0 & 1.8 & 1.4 \\
\hline Rate of return on equity (ROF) & 32.6 & 9.1 & 8.6 & 18.3 & 13.4 \\
\hline Liquid assets (percent) of tolal assets $\mathrm{L}$ & 24.8 & 28.2 & 24.2 & 25.0 & 26.2 \\
\hline \multicolumn{6}{|l|}{ Total domestic private banks } \\
\hline Share in assets & 6.1 & 6.7 & 6.8 & 7.4 & 10.4 \\
\hline Capital adequacy ratio (percent) & 18.1 & 16.6 & 15.2 & 14.8 & 13.6 \\
\hline NPLs (percent) total loans & 4.1 & 6.3 & 7.0 & 6.5 & 8.8 \\
\hline Provisions (percent) NPLs & 31,6 & 31.1 & 25.0 & 30.8 & 18.7 \\
\hline Rate of return on assets (ROA) & 2.6 & 1.0 & 1.0 & 1.2 & 1.1 \\
\hline Fate of retum on equity (ROE) & 22.7 & 9.1 & 8.9 & 13.8 & 14.3 \\
\hline Liquid assets (percent) of total assets $1 /$ & 20.7 & 18.7 & 17.3 & 20.1 & 18.3 \\
\hline \multicolumn{6}{|l|}{ Total system excluding BNF } \\
\hline Share in assets & 85.1 & 86.0 & 88.6 & 90.8 & 92.0 \\
\hline Capital adequacy ratio (percent) & 19.2 & 17.2 & 17.1 & 16.2 & 17.9 \\
\hline NPLs (percent) total loans & 5.6 & 10.3 & 12.0 & 12.3 & 14.7 \\
\hline Provisions (percent) NPLs & 40.3 & 36.5 & 39.2 & 39.8 & 50.2 \\
\hline Rate of retum on assets (ROA) & 4.7 & 2.3 & 1.7 & 2.4 & 1.5 \\
\hline Rate of retum on equity (ROE) & 44.1 & 21.2 & 14.6 & 24.1 & 14.3 \\
\hline Liquid assets (percent) of total assets $1 /$ & 26.9 & 25.3 & 22.3 & 23.2 & 25.7 \\
\hline \multicolumn{6}{|l|}{ National Development Bank (BNF) } \\
\hline Share in assets & 14.9 & 14.0 & 11.4 & 9.2 & 8.0 \\
\hline Capital adequacy ratio (jercent) & 30.0 & 18.4 & 21.2 & 22.9 & 18.8 \\
\hline NPLs (percent) total loans & 44.3 & 49.0 & 44.6 & 46.5 & 56.2 \\
\hline Provisions (percent) NPLs & 25.7 & 33.2 & 38.1 & 31.7 & 39.6 \\
\hline Rate of retum on assets (ROA) & -6.9 & 0.2 & -0.5 & 0.3 & 4.7 \\
\hline Rate of teturn on equity (ROE) & -30.4 & 1.4 & -4.2 & 2.0 & -27.3 \\
\hline Liquid assets (percent) of total assets 1 & 16.1 & 24.4 & 16.7 & 20.4 & 17.3 \\
\hline
\end{tabular}

Source: Superintendency of Banks.

1/ Liquid assets are calculated as the sum af eash, reserves, accounts in banks and lending in interbank market. 
Table 22. Paraguay: Balance of Payments

(In millions of U.S. dollars)

\begin{tabular}{|c|c|c|c|c|c|}
\hline & 1998 & 1999 & 2000 & 2001 & $\begin{array}{r}\text { Prel. } \\
2002 \\
\mathrm{HL} \\
\end{array}$ \\
\hline Current account & -160 & -165 & -150 & -155 & 215 \\
\hline Trade balance & -393 & -441 & .537 & -496 & 29 \\
\hline Exports & 3,548 & 2,310 & 2,326 & 2,463 & 1373 \\
\hline Exports of domestic goods & 1,014 & 741 & 869 & 1,108 & 478 \\
\hline Reexports & 2,530 & 1,566 & 1,453 & 1,355 & 893 \\
\hline Imports & 3,941 & 2,750 & 2,864 & 2,959 & 1344 \\
\hline Registered imports & $\ldots$ & 1,725 & 2,050 & 1,989 & 762 \\
\hline Unregistered imports & $\ldots$ & 1,025 & 813 & 970 & 582 \\
\hline Services (net) & 50 & 82 & 182 & 170 & 119 \\
\hline Transport & -258 & -215 & -167 & -127 & -38 \\
\hline Travel & -31 & -28 & 4 & -14 & $-\mathrm{Il}$ \\
\hline Other & 339 & 326 & 345 & 311 & 169 \\
\hline Factor income & 6 & 18 & 28 & 4 & 19 \\
\hline Transfers & $\cdots$ & 175 & 177 & 167 & 48 \\
\hline Capital and financial account & 325 & 511 & 125 & 313 & -112 \\
\hline Capital transfers & 5 & 20 & 3 & 15 & 8 \\
\hline Direct investment & 336 & 89 & 113 & 74 & 14 \\
\hline Portfolio investment & 9 & -9 & 3 & 0 & 0 \\
\hline Other investrnent & -26 & 411 & 7 & 224 & -134 \\
\hline Assets (increase -) & -5 & -118 & -211 & 208 & -38 \\
\hline Liabilities (increase + ) & -21 & 529 & 217 & 16 & -96 \\
\hline Errors and omissions & -194 & -474 & 208 & -112 & -234 \\
\hline Overall balance & -29 & -129 & 183 & 46 & -132 \\
\hline \multicolumn{6}{|l|}{ Memorandum items: } \\
\hline Current account in percent of GDP & $-1,8$ & -2.1 & -1.9 & -2.3 & $\cdots$ \\
\hline External public debt in percent of GDP & 20.4 & 28.9 & 30.6 & 33.5 & $\cdots$ \\
\hline Debt service in percent of exports GNFS & $\ldots$ & $\ldots$ & 7.0 & 7.8 & $\ldots$ \\
\hline Export Volume (percentage change) & 6.5 & -34.9 & -22.0 & 8.8 & $\ldots$ \\
\hline Import Volume (percentage change) & -6.0 & -30.3 & 0.2 & 2.8 & $\ldots$ \\
\hline Terms of trade (percentage change) & 12.7 & -4.2 & 24.2 & -3.1 & $\ldots$ \\
\hline
\end{tabular}

Sources: Central Bank of Paraguay, and Fund staff estimates. 
Table 23. Paraguay: Composition of Registered Imports

\begin{tabular}{|c|c|c|c|c|c|c|}
\hline & \multirow[b]{2}{*}{1998} & \multirow[b]{2}{*}{1999} & \multirow[b]{2}{*}{2000} & \multicolumn{2}{|c|}{2001} & \multirow{2}{*}{$\begin{array}{r}2002 \\
\mathrm{Hl}\end{array}$} \\
\hline & & & & $\mathrm{Hl}$ & Year & \\
\hline \multicolumn{7}{|c|}{ (In thousands of U.S. dollars) } \\
\hline Total & $2,470,748$ & $1,725,054$ & $2,050,391$ & $1,012,208$ & $1,988,820$ & 761,860 \\
\hline Consumęr goods & $1,203,273$ & 691,228 & $\mathrm{~B} 82,490$ & 438,103 & 833,401 & 304,009 \\
\hline Nondumble goods & $1,010,585$ & 587,357 & 770,846 & 384,296 & 725,592 & 268,285 \\
\hline Food & 155,347 & 78,208 & 139,170 & 64,148 & 124,927 & 45,445 \\
\hline Beverages and tobacco & 429,206 & 220,188 & 205,660 & 94,051 & 163,819 & 54,353 \\
\hline Othes & 426,033 & 288,961 & 426,016 & 226,097 & 436,846 & 168,487 \\
\hline Durable goods & 192,688 & 103,871 & 111,644 & 53,806 & 107,809 & 35,724 \\
\hline Automobiles & 139,675 & 79,031 & 76,777 & 37,189 & 74,721 & 25,587 \\
\hline Electronic equipment & 53,009 & 24,840 & 34,867 & 16,618 & 33,088 & 10,137 \\
\hline Intermediate goods & 512,026 & 419,402 & 610,013 & 307,680 & 636,907 & 223,364 \\
\hline Fuels and lubricants & 188,715 & 194,791 & 312,681 & $160,32 i$ & 302,892 & 100,165 \\
\hline Chemicals & 133.736 & 102,277 & 134,815 & 50,508 & 148,124 & 47,590 \\
\hline Other & 189,575 & 122,334 & 162,517 & 96,850 & 185,891 & 75,609 \\
\hline Capital goods & 755,489 & 614,424 & 557,888 & 266,426 & 518,512 & 234,487 \\
\hline Machinery and motors & 500,051 & 418,613 & 408,487 & 182,714 & 358,354 & 164,720 \\
\hline Transportation equipment & 200,014 & 159,965 & 106,455 & 62,420 & 113,639 & 49,362 \\
\hline Other & 55,424 & 35,846 & 42,946 & 21,293 & 46,519 & 20.404 \\
\hline \multicolumn{7}{|c|}{ (In percent of total registered imports) } \\
\hline Total & 100.0 & 100.0 & 100.0 & 100.0 & 100.0 & 100.0 \\
\hline Consumer goods & 48.7 & 40.1 & 43.0 & 43.3 & 41.9 & 39.9 \\
\hline Nondurable goods & 40.9 & 34.0 & 37.6 & 38.0 & 36.5 & 35.2 \\
\hline Food & 6.3 & 4.5 & 6.8 & 6.3 & 6.3 & 6.0 \\
\hline Beverages and tobaceo & 17.4 & 12.8 & 10.0 & 9.3 & 8.2 & 7.1 \\
\hline Other & 17.2 & 16.8 & 20.8 & 22.3 & 22.0 & 22.1 \\
\hline Durable goods & 7.8 & 6.0 & 5.4 & 5.3 & 5.4 & 4.7 \\
\hline Automobiles & 5.7 & 4.6 & 3.7 & 3.7 & 3.8 & 3.4 \\
\hline Electronic equipment & 2.1 & 1,4 & 1.7 & 1.6 & 1.7 & 1.3 \\
\hline Intermediate goods & 20.7 & 24.3 & 29.8 & 30.4 & 32.0 & 29.3 \\
\hline Fuels and lubricants & 7.6 & 11.3 & 15.2 & 15.8 & 15.2 & 13.1 \\
\hline Chemicals & 5.4 & 5.9 & 6.6 & 5.0 & 7.4 & 6.2 \\
\hline Other & 7.7 & 7.1 & 7.9 & 9.6 & 9.3 & 9.9 \\
\hline Capital goods & 30.6 & 35.6 & 27.2 & 26.3 & 26.1 & 30.8 \\
\hline Machinery and motors & 20.2 & 24.3 & 19.9 & 18.1 & 18.0 & 21.6 \\
\hline Transportatian equipment & 8.1 & 9.3 & 5,2 & 6.2 & 5.7 & 6.5 \\
\hline Other & 2.2 & 2.1 & 2.1 & 2.1 & 2.3 & 2.7 \\
\hline
\end{tabular}

Source: Centrul Bank of Paraguay. 
Table 24. Paraguay: Composition of Registered Exports

\begin{tabular}{|c|c|c|c|c|c|c|}
\hline & \multirow[b]{2}{*}{1998} & \multirow[b]{2}{*}{1999} & \multirow[b]{2}{*}{2999} & \multicolumn{2}{|c|}{2001} & \multirow{2}{*}{$\begin{array}{r}2002 \\
\mathrm{Hl}\end{array}$} \\
\hline & & & & H] & Year & \\
\hline \multicolumn{7}{|c|}{ (In thousands of U.S. dollars) } \\
\hline Total & $1,023,959$ & 753,015 & 877,606 & 508,261 & 999,628 & 480,693 \\
\hline Primary products & 649,835 & 455,268 & 511,617 & 324,054 & 587,215 & 291,949 \\
\hline Agricultural & 541,570 & 382,885 & 382,807 & 258,527 & 450,721 & 215,317 \\
\hline Cotton & 92,050 & 69,136 & 92,528 & 54,191 & 90,505 & 22,231 \\
\hline Soy & 440,315 & 307,135 & 285,924 & 202,357 & 356,315 & 190,281 \\
\hline Other & 9,205 & 6,614 & 4,355 & 1,979 & 3,901 & 2,805 \\
\hline Livestock & 108,265 & 72,383 & 128,810 & 65,527 & 136,494 & 76,632 \\
\hline Meat & 69,462 & 35,394 & 72,728 & 36,588 & 78,091 & 45,026 \\
\hline Leather & 38,803 & 36,989 & 56,082 & 28,939 & 58,403 & 31,606 \\
\hline Manufactured products & 96,115 & 74,480 & 60,278 & 24,842 & 72,833 & 35,503 \\
\hline Alcohol & 925 & 261 & 191 & 3 & 152 & 0 \\
\hline Sugar & 8,931 & 11,986 & 8,057 & 1,895 & 9,270 & 2,855 \\
\hline Oils & 86,259 & 62,233 & 52,030 & 22,944 & 63,411 & 32,648 \\
\hline Wood & 69,657 & 58,797 & 75,062 & 33,579 & 68,738 & 29,100 \\
\hline Other & 208,352 & 164,470 & 230.649 & 125,786 & 270,842 & 124,14 \\
\hline \multicolumn{7}{|c|}{ (In percent of total registered exports) } \\
\hline Total & 100.0 & 100.0 & 100.0 & 100,0 & 100.0 & 100.0 \\
\hline Primary products & 63.5 & 60.5 & 58.3 & 63.8 & 58.7 & 60.7 \\
\hline Agricultural & 52.9 & 50.8 & 43.6 & 50.9 & 45.1 & 44.8 \\
\hline Cotton & 9.0 & 9.2 & 10.5 & 10.7 & 9.1 & 4.6 \\
\hline Soy & 43.0 & 40.8 & 32.6 & 39.8 & 35.6 & 39.6 \\
\hline Other & 0.9 & 0.9 & 0.5 & 0.4 & 0.4 & 0.6 \\
\hline Livestock & 10.6 & 9.6 & 14.7 & 12.9 & 13.7 & 15.9 \\
\hline Meat & 6.8 & 4.7 & 8.3 & 7.2 & 7.8 & 9.4 \\
\hline Leather & 3.8 & 4.9 & 6.4 & 5.7 & 5.8 & 6.6 \\
\hline Manufactured products & 9.4 & 9.9 & 6.9 & 4.9 & 7.3 & 7.4 \\
\hline Alcohol & 0.1 & 0.0 & 0.0 & 0.0 & 0.0 & 0.0 \\
\hline Sugar & 0.9 & 1.6 & 0.9 & 0.4 & 0.9 & 0.6 \\
\hline Oils & 8.4 & 8.3 & 5.9 & 4.5 & 6.3 & 6.8 \\
\hline wood & 6.8 & 7.8 & 8.6 & 6.6 & 6.9 & 6.1 \\
\hline Other & 20.3 & 21.8 & 26.3 & 24.7 & 27.1 & 25.8 \\
\hline
\end{tabular}

Source: Central Bank of Paragouay. 
Table 25. Paraguay: Direction of Trade

(In thousands of U.S. dollars, F. O.B.)

\begin{tabular}{|c|c|c|c|c|c|c|c|c|c|c|c|c|}
\hline \multirow[b]{2}{*}{ Country or Trade Grouping } & \multicolumn{3}{|c|}{1999} & \multicolumn{3}{|c|}{2000} & \multicolumn{3}{|c|}{2001} & \multicolumn{3}{|c|}{2001 (Jan - June) } \\
\hline & Imporss & Exports & Balance & Imports & Exports & Balance & Imporis & Expors & Balance & impors & Exports & Baiance \\
\hline \multicolumn{13}{|l|}{ Mercosur } \\
\hline Argentina & 312,184 & 53,282 & $-258,902$ & 521,384 & 9],998 & $-429,385$ & 478,562 & 60,827 & $-417,735$ & 246,805 & 30,823 & $-215,982$ \\
\hline Brazil & 509,620 & 234,969 & $-274,651$ & 502,072 & $3.36,562$ & $-165,509$ & 563,588 & 277,889 & $-285,699$ & 274,739 & 116,934 & $-157,805$ \\
\hline Uriguay & 68.229 & 19,225 & $-49,005$ & 78,314 & 122,984 & 44,670 & 69,323 & 180,018 & 110,694 & 37,652 & 107,541 & 69,889 \\
\hline \multicolumn{13}{|l|}{ Dther South America } \\
\hline Chile & 4,811 & 23,114 & 18,303 & 32,520 & 49,901 & 17,382 & 42,009 & 61,464 & 19,455 & 22,775 & 27,846 & 5,071 \\
\hline \multicolumn{13}{|c|}{ North and Central America and Caribbean } \\
\hline United States & 235,864 & 57,886 & $-177,979$ & 147,044 & 33,623 & $-113,421$ & 118,392 & 29,327 & $-89,065$ & 60,491 & 10,898 & $-49,594$ \\
\hline Mexica & 10,441 & 1,942 & $-8,499$ & 18,240 & 742 & $-\uparrow 7,498$ & 17,586 & 366 & $-16,820$ & 9,036 & 312 & $-8,725$ \\
\hline Bahamas & 7,381 & 0 & $-7,381$ & 26,919 & 0 & $-26,919$ & 42,783 & 0 & $-42,783$ & 24,716 & 0 & $-24,716$ \\
\hline \multicolumn{13}{|l|}{ Europear Union } \\
\hline Gennany & 57,750 & 11,374 & $-46,376$ & 57,893 & 6,724 & $-51,169$ & 50,264 & 13,056 & $-37,208$ & 23,830 & 5,262 & $-18,568$ \\
\hline Spain & 54,399 & 4.725 & -49.674 & 29,973 & 7,139 & $-22,835$ & 20,990 & 10,848 & $-10,143$ & 12,130 & 5,917 & $-6,213$ \\
\hline Nethertands & 6.587 & 137,136 & 130,549 & 8,812 & 61,685 & 52,873 & 8,784 & 29,378 & 20,594 & 6,531 & 19,690 & {$[3,159$} \\
\hline Italy & 25,117 & 20,075 & $-5,042$ & 44,506 & 30,290 & $-14,216$ & 25,304 & 42,088 & 16,784 & 16,015 & 22,512 & 6,497 \\
\hline United Kingdon & 39,622 & 95,424 & 55,802 & 29,009 & 3,556 & $.25,453$ & 35,106 & 2,557 & $-32,550$ & 21,812 & 1,289 & $-20,523$ \\
\hline \multicolumn{13}{|l|}{ Asia } \\
\hline Japan & 102,687 & 2,186 & $-100,500$ & 92,479 & 2,781 & $.89,697$ & 86,236 & 11,770 & $-74,467$ & 43,422 & 8,641 & $-34,781$ \\
\hline Taiwan Province of China & 121,663 & 19,708 & $-101,955$ & 40,460 & 15,423 & $-25,038$ & 18,454 & 11,648 & $-6,806$ & 9,651 & 5,702 & $-3,949$ \\
\hline India & 6,753 & 0 & $-6,753$ & 6,772 & 1,042 & $-5,731$ & 7,342 & 28,461 & 21,119 & 4,579 & 13,303 & 8,724 \\
\hline \multicolumn{13}{|l|}{ Other } \\
\hline Switzerland & 10,374 & 2,197 & $-8,176$ & 34,659 & 1,638 & $-33,021$ & 36,609 & 34,373 & $-2,237$ & 19,203 & 25,410 & 6,207 \\
\hline Others & 19,506 & 35,684 & 16,178 & 248,952 & 74,637 & $-174,316$ & 251,639 & 146,067 & $-105,572$ & 126,110 & 84,633 & $-41,477$ \\
\hline Tota] & $1,725,054$ & 740,769 & $.984,285$ & $2,050,391$ & 869,357 & $-1,181,034$ & $1,988,820$ & 990,205 & $.998,615$ & $1,012,226$ & 506,364 & $-505,862$ \\
\hline
\end{tabular}

Source: Central Bank of Paraguay. 